\title{
Categorización de las manzanas urbanas para la integración de la silvicultura urbana en la planificación de las ciudades. Caso de estudio: Área Metropolitana de Mendoza
}

\author{
Mariela Edith Arboit ${ }^{1}$, Dora Silvia Maglione ${ }^{2}$ \\ ${ }^{1}$ Instituto de Ciencias Humanas, Sociales y Ambientales (INCIHUSA). Consejo Nacional de \\ Investigaciones Científicas y Técnicas (CONICET), Argentina. ${ }^{2}$ Instituto de Trabajo, Economía y \\ Territorio, Universidad Nacional de la Patagonia Austral, Argentina \\ E-mail: 'marboit@mendoza-conicet.gob.ar, ${ }^{2}$ dmaglione@uarg.unpa.edu.ar
}

\begin{abstract}
Resumen. La urbanización es uno de los principales factores antropogénicos que ha causado la reducción de la superficie verde y la sustitución de los hábitats preexistentes en las ciudades. En la actualidad, más de la mitad de la población humana mundial se concentra en zonas urbanas y la región de américa latina es una de las más urbanizadas del mundo, con el $80 \%$ de su población en las ciudades y un deterioro progresivo en aspectos energético-ambientales.

El objetivo futuro es determinar estrategias de intervención que posibiliten alcanzar mejoras en los valores de actividad fotosintética vegetal en ciudades forestadas de la región insertas en clima seco. El trabajo ha permitido una categorización del índice de vegetación de diferencia normalizada (NDVI) en las manzanas urbanas del Área Metropolitana de Mendoza (AMM) a partir de sistemas de información geográfica (SIG) y del análisis de imágenes satelitales Landsat 8.

Los resultados obtenidos indican un vigor vegetativo nulo o bajo del $39.87 \%$ en las manzanas del AMM y moderado del $45.47 \%$. La categorización de las manzanas, ha permitido además un análisis estadístico preliminar por departamento considerando las estaciones otoño-invierno y primavera-verano. En el AMM el NDVI anual del 52.08\% de las manzanas urbanas de Godoy Cruz se ubican en el rango nulo o bajo; seguido por Guaymallén $48.24 \%$ y Maipú 45.61\%. La gobernanza eficiente de las ciudades en la región requiere de políticas proactivas, planificación estratégica y legislación que integren la silvicultura urbana. El trabajo aporta conocimiento de base, necesario para un enfoque integrado de sostenibilidad.
\end{abstract}

Palabras clave: Vegetación, SIG, teledetección.

\section{Introducción}

Importantes contribuciones conceptuales sobre aspectos generales y específicos del desarrollo urbano sostenible, cobran particular relevancia ante el constante crecimiento que va incrementando la población mundial, el consumo de los recursos no renovables y la tasa de urbanización en países en desarrollo y emergentes. Las teorías más significativas de diferentes autores enmarcan los conceptos y principios de la sostenibilidad y el desarrollo sostenible, la antinomia entre crecimiento y desarrollo, las aproximaciones conceptuales a la problemática de la sostenibilidad: sostenibilidad fuerte y sostenibilidad débil, los modelos teóricos de ciudades sostenibles: la ciudad compacta y la ciudad difusa, las interpretaciones desde el contexto de los países en desarrollo y los objetivos a nivel mundial de ciudades inclusivas, seguras, resilientes y sostenibles; parte integrante de la discusión 
durante la reunión de Hábitat III donde los países adoptaron por unanimidad la Nueva Agenda Urbana con acciones concretas para asegurar que el desarrollo urbano sea gestionado de manera más sostenible y ambientalmente sano. A fin de lograr los objetivos de la ciudad sostenible, numerosos trabajos han estudiado la incorporación de la forestación como una forma de mitigación de las condiciones climáticas (Santamouris et al, 2018; McPherson et al, 2017). La incorporación de la cobertura vegetal juega un importante rol en el ahorro energético y el mejoramiento de la calidad del ambiente urbano, con grandes beneficios como el efecto de "isla fría diurna" observado en regiones áridas.

Metodológicamente la teledetección desde los años 70 (Monteith, 1973) contribuyó con los primeros estudios. Recientemente, los mapeos de la cobertura vegetal han sido relacionados a escala urbana con: microclima (Davis et al, 2016), uso del suelo urbano (Gandhi, Parthiban y Thummalu, 2015); crecimiento urbano, superficies impermeables, clasificación de ciudades verdes (Gärtner, 2017), efecto oasis e impacto de la urbanización en ciudades subtropicales del desierto (Fan et al, 2017), identificación de la infraestructura verde y su distribución en relación a parámetros administrativos y urbanísticos en ciudades.

Se avanza, en el presente trabajo con la categorización de las manzanas urbanas a partir del estudio del NDVI, con el objetivo de conocer las condiciones actuales de vegetación del AMM, a fin de integrar la silvicultura urbana en la planificación de la ciudad.

\section{Situación de Referencia}

La zona definida como universo de análisis es el AMM ubicada en el centro-oeste argentino (latitud: -32.89 ; longitud: -68.83 , altitud: 827 m.s.n.m.), en el oasis agrícola norte la provincia de Mendoza (Figura 1), al pie de la Cordillera de los Andes. Es la cuarta aglomeración urbana argentina, cuenta con una población de 937.145 habitantes y ocupa una extensión de $252 \mathrm{~km} 2$, conformada por la ciudad de Mendoza Capital y cinco departamentos limítrofes: Las Heras, Godoy Cruz, Guaymallén, Luján de Cuyo y Maipú (Figura 2).

\section{Metodología}

El desarrollo metodológico de esta investigación ha sido ya parcialmente informado en publicaciones previas (Arboit y Maglione, 2018), se estima necesario presentar en la figura 3 una síntesis del mismo con el fin de facilitar la comprensión de los resultados de las tareas recientes que se presentan por primera vez en este trabajo.

\section{La labor realizada se desglosa en:}

1. Recopilación de la información cartográfica disponible en entorno GIS para el AMM. Análisis de: imágenes satelitales mensuales provenientes de Landsat 8 para el período 2013-2017 (United States Geological Survey, 2016), datos catastrales urbanoedilicios (Dirección Provincial de Catastro, 2010). Preparación de mapas base para el AMM.

\begin{tabular}{|c|c|c|c|c|c|c|}
\hline \multirow[t]{8}{*}{ Oasis Norte (Rios Meñozaz Y Tunuyant tramo inferior) } & \multirow{8}{*}{ A } & \multirow[t]{2}{*}{ Departamento } & \multicolumn{2}{|c|}{ Hab.Urbanos $\left(\mathrm{N}^{0}\right)$} & \multicolumn{2}{|c|}{ Sup.Urbana(Ha) } \\
\hline & & & 1991 & 2010 & 1990 & 2017 \\
\hline & & CAPITAL & 121620 & 114893 & 23.81 & 32.73 \\
\hline & & GODOY CRUZ & 179553 & 191299 & 27.06 & 35.30 \\
\hline & & GUAYMALLEN & 200477 & 252618 & 35.17 & 102.87 \\
\hline & & LAS HERAS & 145680 & 189067 & 25.85 & 71.49 \\
\hline & & LUJAN & 54210 & 82615 & 15.55 & 78.14 \\
\hline & & MAIPU & 71603 & 106662 & 16.87 & 85.21 \\
\hline $\begin{array}{llr}\text { Figura 1: Ubicación del } \\
\text { Área Metropolitana de } \\
\text { Mendoza. Ref: Martí, L. }\end{array}$ & \multicolumn{6}{|c|}{$\begin{array}{l}\text { Figura 2: Área Metropolitana de Mendoza. Ubicación de los } \\
\text { departamentos administrativos, habitantes urbanos y superficie } \\
\text { urbana. Ref: Arboit, M; Indec; Molina, G. y Sedevich, A. }\end{array}$} \\
\hline
\end{tabular}




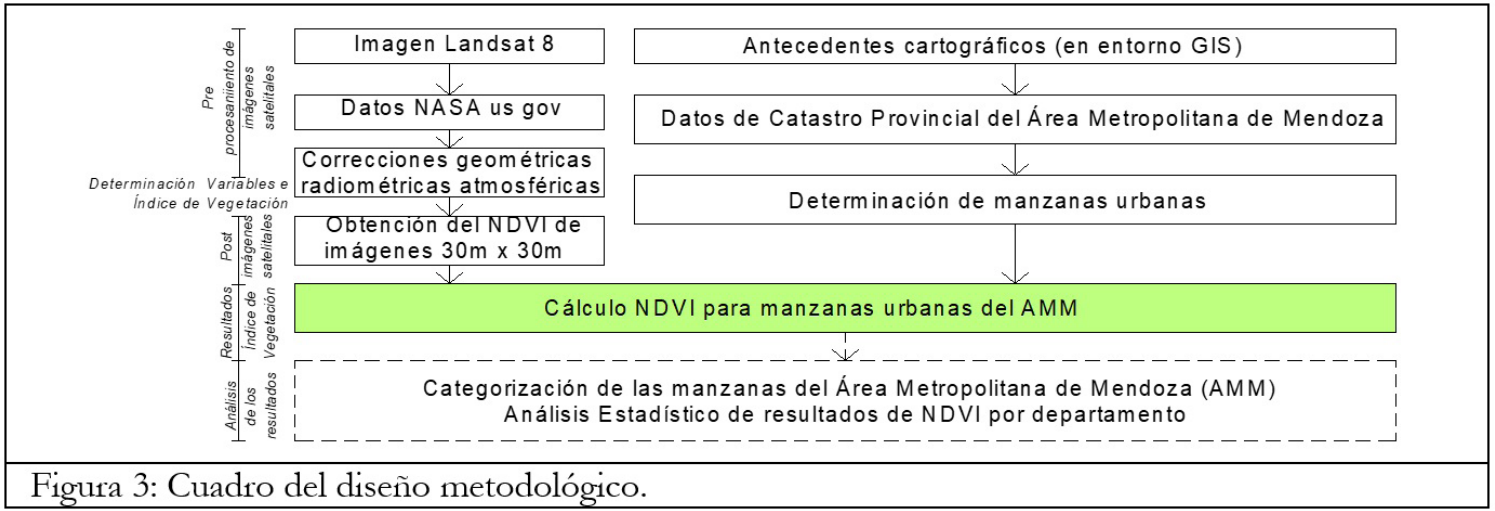

2. Selección del indicador descriptivo de la actividad fotosintética de la infraestructura verde a escala urbana.

Índice de Vegetación deDiferencia Normalizada (NDVI): es el cociente normalizado entre bandas espectrales (rojo e infrarrojo cercano) registradas por el sensor satelital Operational Land Imager (OLI), con una resolución espacial de 30 metros para las Bandas 4 y 5 (Earth Observation Group, 2017), el índice permite identificar la vegetación de otras superficie; varía entre $-1 \mathrm{y}+1$ : valores altos revelan alta actividad fotosintética de la cubierta vegetal (fenología foliar) y estrecha relación con la evapotranspiración; valores bajos indican situaciones de escasa o nula cubierta vegetal y baja actividad fotosintética; valores negativos corresponden principalmente a nubes, agua y nieve. La siguiente ecuación responde al índice (Rouse et al, 1974):

$$
\begin{aligned}
N D V I= & (N I R-R) \div(N I R+R) \\
\text { Dónde: } & \mathrm{NIR}=\text { Infrarrojo Cercano } \\
& \mathrm{R}=\text { Rojo }
\end{aligned}
$$

Se realizó el cálculo y mapeo de los valores NDVI para 10390 manzanas urbanas, considerando 38 escenas mensuales en el periodo 2013-2017 (394820 resultados para el período 2013-2017), concluyendo en este punto la exposición de la labor ya informada (Arboit y Maglione, 2018).

Se toma a la manzana urbana como unidad de análisis. Se considera la superficie de la manzana urbana desde eje de calle a eje de calle en el cálculo del NDVI a fin de incluir el arbolado urbano. Se relacionan los valores de índice al límite interior de la manzana.
Los valores analizados son los promedios por departamento de todas las unidades de análisis.

3. Categorización de las manzanas urbanas en función de una escala de valores medios de NDVI.

4. Análisis estadístico. Conocidos los niveles de actividad fotosintética a través del NDVI por manzana, se analizan los resultados anuales y estacionales.

\section{Resultados}

Manzanas Urbanas del AMM. El índice NDVI se ha calculado y cartografiado para cada manzana urbana del AMM por departamento administrativo.

El valor NDVI considera la superficie de la manzana urbana desde eje de calle a eje de calle, posteriormente se ha relacionado dicho valor al límite interior de la manzana, de esta forma la cartografía permite visualizar las zonas más comprometidas desde el punto de vista del vigor vegetativo. Del total de manzanas analizadas 10390, se han considerado 10332 para el estudio y 38 escenas para cada una de las manzanas (392616 resultados para el período 2013-2017). En las figuras 4 y 5 se presenta la cartografía de dos escenas elegidas como representativas (estival e invernal).

El análisis de los resultados indica un valor promedio NDVI para la totalidad de las manzanas urbanas de 0.241 .

Considerando las manzanas urbanas por departamento, Lujan de Cuyo posee el valor medio más alto (0.29), seguido por Capital (0.25), Maipú (0.24) y Las Heras (0.24). Los departamentos que presentan valores medios más bajos son Guaymallén (0.22); y Godoy 


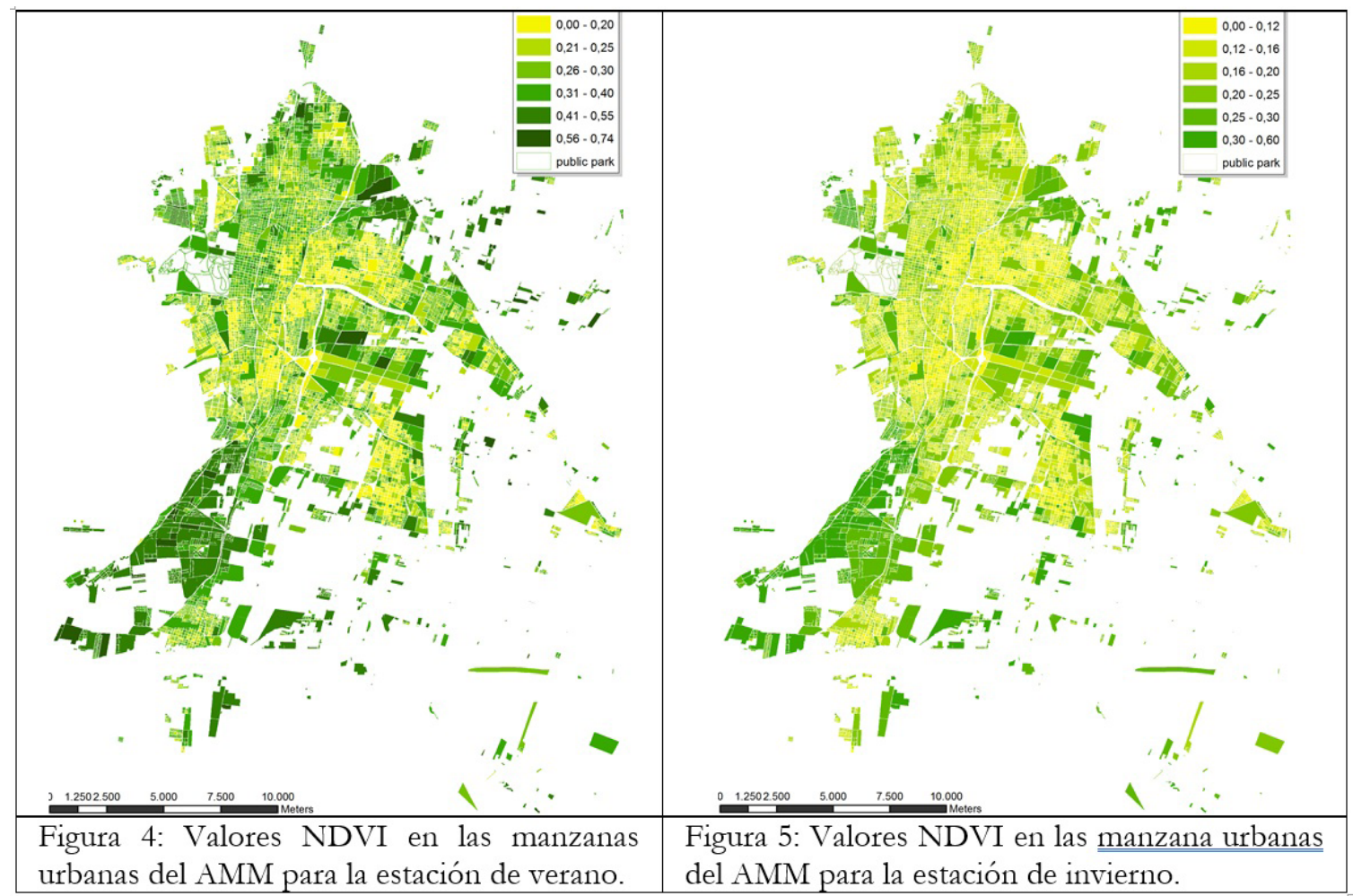

Cruz (0.21). El NDVI se ve explicado por la cobertura forestal (arbolado público y privado), por la cobertura vegetal a nivel de suelo (superficies parquizadas) y en los demás estratos vegetales. El test LSD de Fisher indica que el valor medio NDVI de las manzanas urbanas de Lujan de Cuyo es significativamente distinto al del resto de las manzanas del AMM (Figura 6 y Tabla 1).

De la comparación del NDVI entre las manzanas urbanas se desprende que los índices NDVI tienen una correlación positiva muy alta considerando los diferentes departamentos (Tabla 2).

\section{Categorización de las manzanas urbanas.}

En la figura 7 se han categorizado las manzanas urbanas en cinco tipologías en función de los valores medio anuales de NDVI: 0.000.20 (bajo o nulo vigor vegetativo); 0.21 0.30 (moderado vigor vegetativo); $0.31-0.40$ (medio vigor vegetativo); $0.41-0.50$ (alto vigor vegetativo); más de 0.51 (muy alto vigor vegetativo), (Figura 8). Al analizar el porcentaje de manzanas urbanas por categoría y por departamento el $52.08 \%$ de las manzanas urbanas de Godoy Cruz se ubican en el rango menor; seguidas por las manzanas urbanas de
Tabla 1: NDVI. Test: LSD Fisher. Fuente: Elaboración.

\begin{tabular}{|l|c|c|c|c|c|}
\hline Departamento & Medias & & & & \\
\hline G. Cruz & 0.21 & A & & & \\
\hline Guaymallén & 0.22 & A & B & & \\
\hline Maipú & 0.24 & & B & C & \\
\hline Las Heras & 0.24 & & B & C & \\
\hline Capital & 0.25 & & & C & \\
\hline L. Cuyo & 0.29 & & & & D \\
\hline
\end{tabular}

Tabla 2: Correlación de Pearson:Coeficientes \probabilidades. Fuente: Elaboración propia.

\begin{tabular}{|l|c|c|c|c|c|l|}
\hline & Capital & G.Cruz & Guaymallén & L.Heras & L.Cuvo & Maipú \\
\hline Capital & & $<0.000$ & $<0.000$ & $<0.000$ & $<0.000$ & $<0.000$ \\
\hline G. Cruz & $\mathbf{0 . 9 9 2}$ & & 0.002 & $<0.000$ & $<0.000$ & $<0.000$ \\
\hline Guaymallén & $\mathbf{0 . 9 9 0}$ & $\mathbf{0 . 9 9 2}$ & & $<0.000$ & $<0.000$ & $<0.000$ \\
\hline Las Heras & $\mathbf{0 . 9 9 0}$ & $\mathbf{0 . 9 8 7}$ & $\mathbf{0 . 9 9 4}$ & & $<0.000$ & $<0.000$ \\
\hline L. Cuyo & $\mathbf{0 . 9 7 1}$ & $\mathbf{0 . 9 7 6}$ & $\mathbf{0 . 9 7 7}$ & $\mathbf{0 . 9 6 9}$ & & $<0.000$ \\
\hline Maipú & $\mathbf{0 . 9 0 9}$ & $\mathbf{0 . 9 1 2}$ & $\mathbf{0 . 9 2 9}$ & $\mathbf{0 . 9 2 9}$ & $\mathbf{0 . 9 3 2}$ & \\
\hline
\end{tabular}



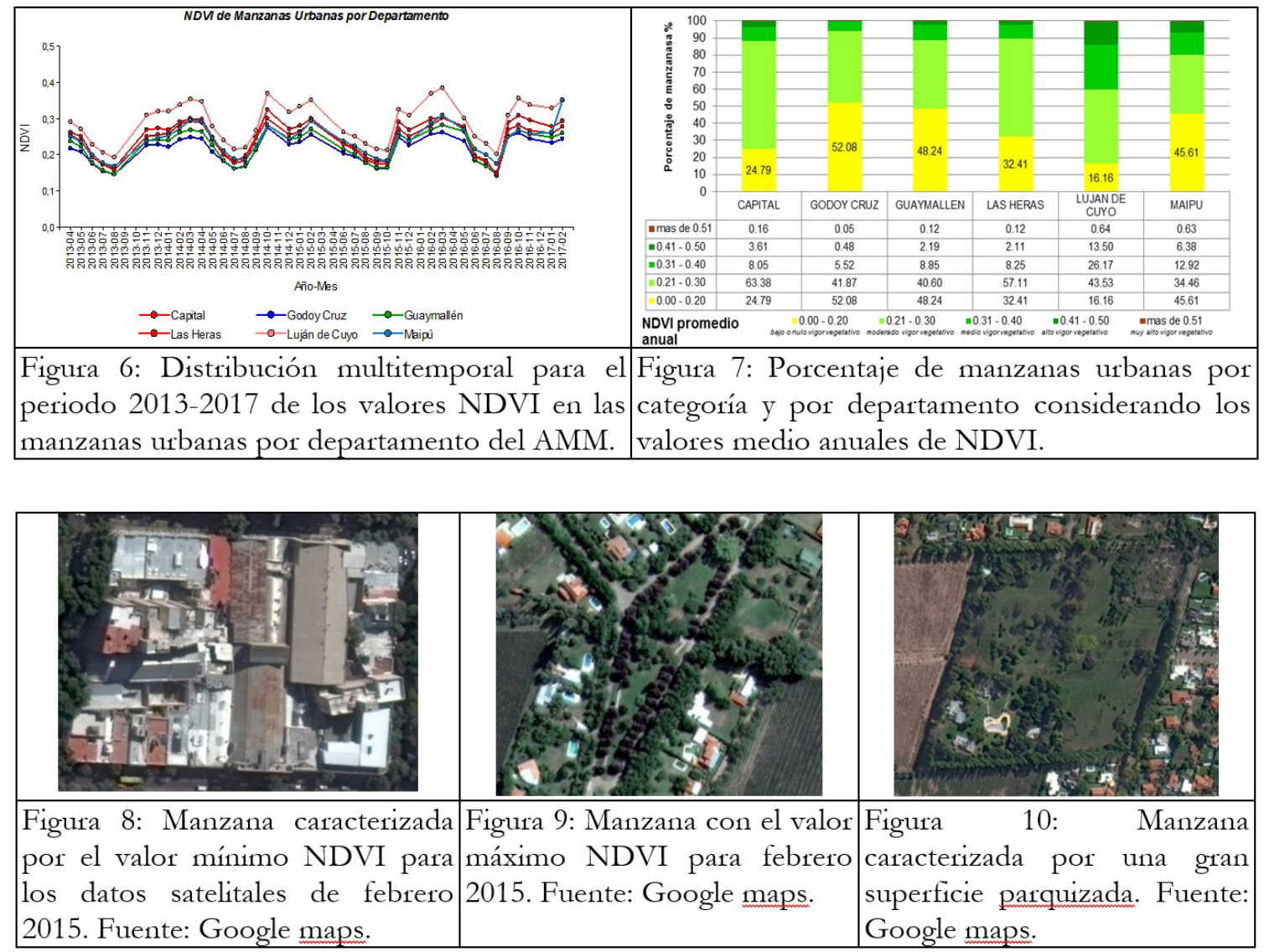

Guaymallén $48.24 \%$ y Maipú 45.61\%. Esto representa un vigor vegetativo nulo o muy bajo para el $39.87 \%$ del total de las manzanas del AMM. El $45.47 \%$ de las manzanas urbanas del AMM resultaron con moderado vigor vegetativo.

Considerando los valores medios mensuales de cada escena por departamento, las manzanas con mayor valor NDVI se ubican en Luján de Cuyo 0.384 para marzo de 2016, el menor valor medio fue 0.140 que se ubicó en Capital para el mes de agosto 2016. Los resultados muestran diferencias significativas entre manzanas. $\mathrm{Si}$ se estudian los valores unitarios por manzana, el valor máximo calculado fue de 0.792 en septiembre de 2014.

En la cartografía para la escena de febrero 2015 (Figura 5), el valor mínimo calculado fue de 0.079 en verano y 0.078 en invierno correspondiente a la manzana urbana ubicada en el microcentro entre las calles San Juan, Buenos Aires, Lavalle y Av. San Martin (Figura 8), otras manzanas vecinas con alta densidad edilicia y sellamiento de suelo, tienen valores similares. El valor máximo NDVI para la misma escena fue de 0.74 correspondiente a una manzana ubicada en Jardines de Santa Oliva Ruta Panamericana 82 y José Hernández, ubicada en un entorno agrícola periurbano (Figura 9). Otro ejemplo tipológico de manzana, ubicada en el departamento de Lujan se muestran en la figura 10 , con valores medios NDVI de 0.58 para febrero 2015 y 0.35 para julio 2014.

\section{Análisis preliminar estacional del NDVI (primavera-verano/otoño-invierno) para el período 2013-2017.}

Parte del comportamiento está determinado por las condiciones climáticas, además una serie de variables influyen en los ciclos fenológicos y de desarrollo estacional que determina oscilaciones naturales de la vegetación con cambios en la distribución temporal y espacial durante los meses del año (Figuras 11 a 12).

Los resultados indican valores medios de NDVI con diferencias marcadas en las 

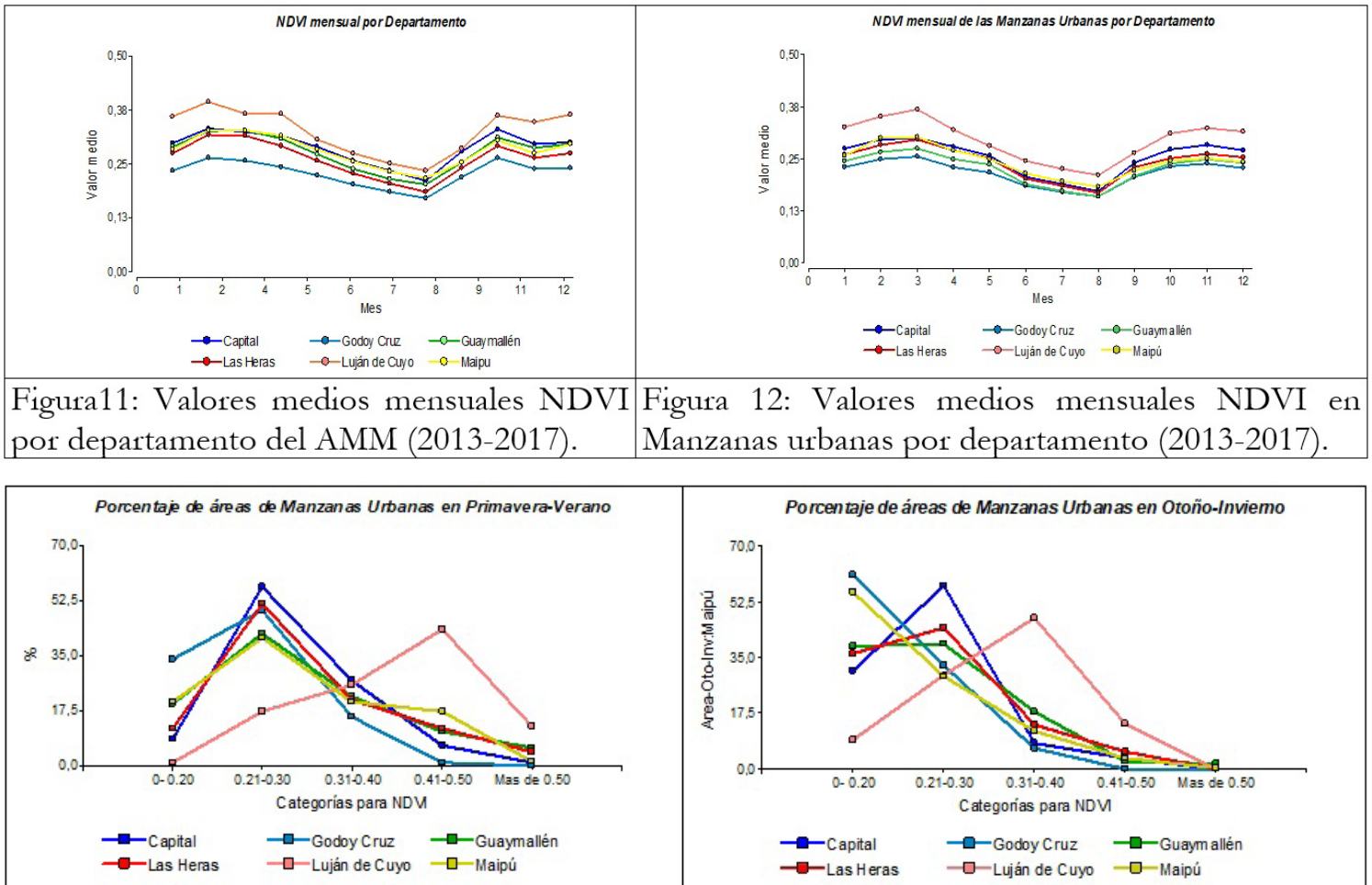

Figura 13: Porcentaje del área de las manzanas Figura 14: Porcentaje del área de las manzanas urbanas por categoría y por departamento urbanas por categoría y por departamento considerando los valores NDVI para la estación considerando los valores NDVI para la estación primavera-verano. otoño-invierno.
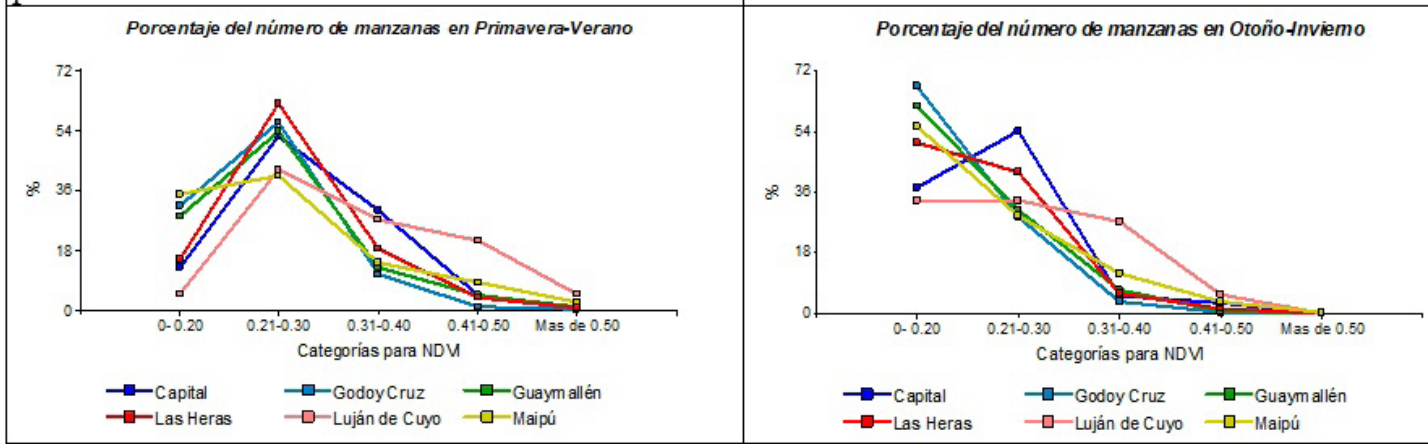

Figura 15: Porcentaje del número de las Figura 16: Porcentaje del número de las manzanas urbanas por categoría y por manzanas urbanas por categoría y por departamento considerando los valores NDVI departamento considerando los valores NDVI para la estación primavera-verano. para la estación otoño-invierno.

estaciones de invierno y verano. Se ofrece los gráficos con los valores obtenidos considerando el área y el número de manzanas (Figuras 1316).

En la estación de primavera-verano el porcentaje de áreas que prevalece en los departamentos es de un moderado vigor vegetativo (NDVI 0.21-0.30) salvo en Luján de Cuyo donde predomina un alto vigor vegetativo (NDVI 0.41-0.50) en el $43.43 \%$ del área ocupada por las manzanas urbanas y el $98.98 \%$ del áreas de manzanas con un vigor vegetativo de moderado a muy alto. $\mathrm{Si}$ se considera el porcentaje de la cantidad de manzanas en Luján el (42.06\%) se ubicó entre 0.21 y 0.30 (Figura 15).

Capital posee un $91.60 \%$ del área de las manzanas con un vigor vegetativo de moderado a muy alto, de dicho porcentaje el $56.94 \%$ es moderado (NDVI $0.21-0.30$ ) y $27.14 \%$ medio 
(NDVI entre 0.31-0.40).

Para la misma estación en el departamento de Godoy Cruz se observa el menor porcentaje del área de manzanas con vigor vegetativo de moderado a muy alto el $66.15 \%$, de las cuales el $49.50 \%$ es un vigor vegetativo moderado. Godoy Cruz y Maipú son los departamentos con mayor cantidad de manzanas y áreas de manzanas con bajo o nulo vigor vegetativo (NDVI 0.00- 0.20), el 33.85\%del área de manzanas de Godoy Cruz y $34.92 \%$ de las manzanas de Maipú. Estos valores tienen una incidencia fundamental de primer orden en la planificación de estrategias para el aumento futuro de los valores del índice de vegetación.

En otoño-invierno, el porcentaje de manzanas con categoría de bajo-nulo vigor vegetativo es superior a la de primavera-verano en todas las localidades. En la estación de otoño-invierno se deberían priorizar especies forestales caducifolias (máxima trasmisividad de la irradiancia solar) y de bajo consumo hídrico (coberturas superficiales de tipo C. dactylon), que se ven asociadas a bajos niveles de vigor vegetativo para dicha estación.

Analizando los resultados anuales el vigor vegetativo de Luján de Cuyo es el mejor en comparación con el resto de los departamentos, con un NDVI $>0.20$ del $95.13 \%$ del área de las manzanas en la estación de primavera-verano y $<0.21$ del $33.61 \%$ en otoño-invierno.

Godoy Cruz es el departamento con valores menores en otoño-invierno, el $67.62 \%$ del área de las manzanas poseen NDVI $<0.21$. En la estación de primavera-verano estos valores no alcanzan a recuperarse, observándose el $68.73 \%$ del área de las manzanas con valores NDVI $>0.20$, ocupando el segundo lugar en orden ascendente después de Maipú $65.08 \%$, para dicha estación.

Los departamentos que más recuperan el vigor vegetativo en el área de manzanas entre las estaciones de otoño-invierno y primaveraverano son Lujan de Cuyo y Capital.

Luján de Cuyo presenta una curva ascendente en relación al porcentaje de áreas de manzanas urbanas (desde valores de 0.000.20 hasta valores de $0.31-0.40$ ) en otoñoinvierno y (desde 0.00- 0.20 hasta 0.41-0.50) en primavera-verano (Figuras 13 y 14); en contraste con los demás departamentos. Especialmente Godoy Cruz y Maipú que presentan la curva descendente en la estación de otoño-invierno es decir el mayor porcentaje de manzanas se ubican entre 0.00 y 0.20 que representa un bajo o nulo vigor vegetativo, mientras el menor porcentaje en manzanas presentan valores mayores a 0.50 (Figura 14).

Se detecta asociación entre la categoría de NDVI y la estación del año en cada departamento, para ambas variables: porcentaje del área de las manzanas urbanas y porcentaje del número o cantidad de las manzanas urbanas (Tabla 3). Los valores significativos en la tabla 3 se dan por la diferencia en las dos primeras categorías, salvo en Luján de Cuyo donde las

Tabla 3: p-valor por departamento. Fuente: Elaboración propia.

\begin{tabular}{|l|c|c|}
\hline Departamento & \% de Área (p-valor) & $\%$ Cantidad de Manzanas (p-valor) \\
\hline Capital & $<0.0001$ & $<0.0001$ \\
\hline Godoy Cruz & 0.0011 & $<0.0001$ \\
\hline Guaymallén & 0.0050 & $<0.0001$ \\
\hline Las Heras & 0.0003 & $<0.0001$ \\
\hline Luján de Cuyo & $<0.0001$ & $<0.0001$ \\
\hline Maipú & $<0.0001$ & 0.0462 \\
\hline
\end{tabular}

Tabla 4: p-valor por estación. Fuente: Elaboración propia.

\begin{tabular}{|l|c|c|}
\hline Departamento & \% de Área (p-valor) & $\%$ Cantidad de Manzanas (p-valor) \\
\hline Otoño-invierno & $<0.0001$ & $<0.0001$ \\
\hline Prima-vera verano & $<0.0001$ & $<0.0001$ \\
\hline
\end{tabular}


diferencias se dan en categorías más elevadas (Figuras 13 a 16).

El porcentaje de área de cada categoría NDVI depende de cada departamento, tanto en otoño-invierno como en primavera-verano (Tabla 4).

\section{Conclusiones}

La investigación profundizó el estudio de la actividad fotosintética vegetal en las manzanas urbanas del AMM a través del índice NDVI y propuso una categorización útil para identificar de manera sencilla, las manzanas que poseen de nulo a muy alto vigor vegetativo. Fue posible además elaborar una base de datos y documentos cartográficos de consulta para los responsables de implementar políticas proactivas, planificación estratégica y legislación.

El análisis estadístico estacional reveló que en la estación primavera-verano los departamentos que poseen mayor número de manzanas con un bajo o nulo vigor vegetativo son: Maipú (34.92\%), Godoy Cruz (31.27\%) y Guaymallén (28.03\%); para la misma estación los departamentos con mayor cantidad de áreas de manzanas a intervenir son Godoy Cruz (33.85\%), seguido por Maipú (20.25\%) y Guaymallén (19.54\%). En la estación de otoño-invierno Godoy Cruz es el departamento con menores valores de NDVI considerando el área de las manzanas.

El análisis anual de los resultados para el período de estudio 2013-2017, determinó que Godoy Cruz es el departamento prioritario a considerar en las estrategias de planificación y mejora de los índices de vegetación, seguido por las manzanas urbanas de Guaymallén, ambos departamentos poseen el mayor número de habitantes urbanos del AMM, con los valores medios más altos de huella y densidad edilicia después de Capital.

Maipú, Guaymallén y Las Heras deberían priorizar la estrategia de aumento de arbolado y superficies vegetadas, en el ámbito público y privado (fuera y dentro de la línea de edificación municipal) a fin de mitigar la tendencia significativa descendente en la actividad fotosintética vegetal en el período 1986-2011 (Arboit y Maglione, 2018), la mayor variación absoluta en cantidad de habitantes a escala provincial (período 2001-2010) y el gran crecimiento de la mancha urbana.

Las manzanas urbanas donde debería implementarse una estrategia de tutela y conservación de la vegetación se ubican en Lujan de Cuyo, dichas manzanas son significativamente distintas al resto de las manzanas del AMM. Luján de Cuyo debe tomar acciones concretas a fin de salvaguardar su vegetación, considerando que es el departamento del AMM con mayores posibilidades a la hora de intervenir con políticas públicas (menor cantidad de habitantes y sellamiento de suelo) pero amenazado hoy por el crecimiento demográfico y la expansión urbana que podrían impactar negativamente en el índice de vegetación.

Las manzanas de Capital ocupan el segundo lugar en orden descendente considerando los valores anuales NDVI, aun siendo el departamento con menor superficie urbana, mayor densidad edilicia, mayor sellamiento de suelo y mayor número de habitantes por kilómetro cuadrado después de Godoy Cruz. En este caso, los esfuerzos de silvicultores urbanos, gestores públicos y privados por el mantenimiento del arbolado, acequias, sistema de riego y espacios verdes se refleja en los resultados alcanzados. Capital debería priorizar además de la tutela y conservación de la vegetación existente; la estrategia de aumento de cobertura vegetal pública y privada. En este sentido, es fundamental la incorporación de nuevas infraestructuras verdes como cubiertas y muros vegetados en las manzanas donde el arbolado urbano no alcanza a compensar el impacto antrópico y edilicio sobre el índice de vegetación.

Las ciudades en la región demandan actores comprometidos con la silvicultura urbana para alcanzar mejoras en los valores de actividad fotosintética vegetal en ciudades forestadas de la región insertas en clima seco.

\section{Agradecimientos:}

El desarrollo de esta investigación contó con el financiamiento de los proyectos PPI-FAUD-Universidad de Mendoza; PIP11220130100407 (CONICET); UE CONICET 
2017- 2022/22920170100036. Algunas imágenes han sido procesadas y elaboradas con la colaboración de M. Fontanive (Politecnico di Torino), C. Cucchietti y J. Morales (Universidad de Mendoza), como parte de prácticas profesionales supervisadas realizadas en INCIHUSA-CONICET.

\section{Referencias}

Arboit, Mariela y Maglione, Dora. 2018. "Situación actual y cambios recientes en los índices de vegetación (VIS) en ciudades forestadas con climas secos. Caso área metropolitana de Mendoza, Argentina". Revista Urbano. Universidad del Bío-Bío, vol.38, pp. 18-35.

Davis, Amelie; Jung, Jinha; Pijanowski, Bryan y Minor, Emily. 2016. "Combined vegetation volume and greenness affect urban air temperatura". Applied Geography, vol. 71, pp. 106-114.

Dirección Provincial de Catastro [en línea]. [Consultado 1 junio 2010]. https:www. atm.mendoza.gov.ar/portalatm/zoneTop/ catastro/catastro.jsp

Earth Observation Group (EOG). NOAA National Geophysical Data Center [en línea]. [Consultado 15 enero 2017]. https:// ngdc.noaa.gov/eog/night_sat/nightsat.html

Fan, Chao; Myint, Soe W.; Kaplan, Shai; Middel, Ariane; Zheng, Baojuan; Rahman, Atiqur; Huang, Huei-Ping; Brazel, Anthony y Blumberg, Dan G. 2017. "Understanding the impact of urbanization on surface urban heat islands-A longitudinal analysis of the oasis effect in subtropical desert cities". Remote Sensing, vol. 9, nº 7, pp. 672.

Gandhi, Meera; Parthiban, S. y Thummalu, C. 2015. "Ndvi: Vegetation change detection using remote sensing and gis - A case study of Vellore District". Procedia Computer Science, vol. 57, pp. 1199-1210.

Gärtner, Philipp. European capital greenness evaluation [en línea]. [Consultado 22 agosto 2017]. https://philippgaertner.github. io/2017/10/ european-capital-greennessevaluation/

McPherson, E. Gregory; Xiaob, Qingfu; van Doornc, Natalie S.; de Goeded, John; Bjorkmand, Jacquelyn; Hollanderd, Allan;
Boyntond, Ryan; Quinnd, James y Thorne, James. 2017. "The structure, function and value of urban forests in California communities". Urban Forestry \& Urban Greening, vol. 28, pp. 43-53.

Monteitn, John. 1973. Principles of Environmental Physics. Londres: Edward Arnold.

Rouse, John; Haas, R.; Deering, D., Schell, J. y Harlan, J. 1974. Monitoring the vernal advancement and retrogradation (Green wave effect) of natural vegetation, Final Report. Texas: A \& M Universiy.

Santamouris, Mattheos; Haddad, Shamila; Saliari, Maria; Vasilakopoulou, Konstantina; Synnefa, Afroditi; Paolini, Riccardo; Ulpiani, Giulia; Garshhasbi, Samira y Fiorito, Francesco. 2018. "On the energy impact of urban heat island in Sydney. Climate and energy potential of mitigation technologies". Energy and Buildings, vol. 166, pp. 154-164.

United States Geological Survey [en línea]. [Consultado 20 septiembre 2016]. Disponible en: https://earthexplorer.usgs. gov/ 